\title{
Association of a higher density of specialist neuroscience providers with fewer deaths from stroke in the United States population
}

\author{
Clinical article
}

\author{
Atman Desai, M.D., ${ }^{1}$ Kimon Bekelis, M.D., ${ }^{1}$ Wenyan Zhao, Рh.D., ${ }^{2}$ \\ Perry A. Ball, M.D., ${ }^{1-3}$ and Kadir Erkmen, M.D. ${ }^{1,2,4,5}$
}

Sections of ${ }^{1}$ Neurosurgery and ${ }^{3}$ Critical Care Medicine,${ }^{4}$ Department of Neurology, and ${ }^{5}$ Cerebrovascular Disease and Stroke Program, Dartmouth-Hitchcock Medical Center, Lebanon; and ${ }^{2}$ Dartmouth Medical School, Hanover, New Hampshire

\begin{abstract}
Object. Stroke is a leading cause of death and disability. Given that neurologists and neurosurgeons have special expertise in this area, the authors hypothesized that the density of neuroscience providers is associated with reduced mortality rates from stroke across US counties.

Methods. This is a retrospective review of the Area Resource File 2009-2010, a national county-level health information database maintained by the US Department of Health and Human Services. The primary outcome variable was the 3-year (2004-2006) average in cerebrovascular disease deaths per million population for each county. The primary independent variable was the combined density of neurosurgeons and neurologists per million population in the year 2006. Multiple regression analysis was performed, adjusting for density of general practitioners (GPs), urbanicity of the county, and socioeconomic status of the residents of the county.

Results. In the 3141 counties analyzed, the median number of annual stroke deaths was 586 (interquartile range [IQR] 449-754), the median number of neuroscience providers was 0 (IQR 0-26), and the median number of GPs was 274 (IQR 175-410) per million population. On multivariate adjusted analysis, each increase of 1 neuroscience provider was associated with 0.38 fewer deaths from stroke per year $(\mathrm{p}<0.001)$ per million population. Rural location $(\mathrm{p}<0.001)$ and increased density of GPs $(\mathrm{p}<0.001)$ were associated with increases in stroke-related mortality.

Conclusions. Higher density of specialist neuroscience providers is associated with fewer deaths from stroke. This suggests that the availability of specialists is an important factor in survival after stroke, and underlines the importance of promoting specialist education and practice throughout the country.
\end{abstract} (http://thejns.org/doi/abs/10.3171/2012.10.JNS12518)

KeY WoRDs $\bullet$ stroke $\bullet$ Area Resource File database $\bullet$
neurologist density $\bullet$ neurosurgeon density $\quad$ vascular disorders

$\mathrm{S}$ TROKE is among the leading causes of death and disability in the US. ${ }^{3,5}$ Health care costs for patients with stroke present a significant economic burden $^{10,14}$ due to several factors, including prolonged hospitalizations, inpatient and outpatient rehabilitation, and long-term care requirements..$^{10,14}$ Although the contribution of GPs in improving health care outcomes has been widely recognized, the impact of specialist care on public

Abbreviations used in this paper: ACA = Affordable Care Act; $\mathrm{ARF}=$ Area Resource File; $\mathrm{CL}=$ confidence level; $\mathrm{GP}=$ general practitioner; IQR = interquartile range . health improvement, particularly the impact of neuroscience providers, has not been investigated.?

One feature of the ACA, which was passed in May 2010, was the incentives for primary care to improve public health care outcomes. Specialist neuroscience providers, including neurologists and neurosurgeons, are currently not provided with the same incentives under the ACA, despite having a potentially large effect on outcomes after stroke. A recent study using the ARF, a national health resource information database, demonstrated that increased density of general surgeons is associated with a reduced mortality rate from motor vehicle 
crashes, ${ }^{7}$ probably as a result of the availability of acute trauma teams. Given that strokes are a similarly frequent cause of death, and neurologists and neurosurgeons hold special expertise in this area relative to other members of the health care team, we postulated that the density of neurosurgeons and neurologists within a given geographical area would be related to reduced numbers of deaths from stroke. The purpose of the present study was to investigate this hypothesis and to quantify any relationship between the availability of specialist neuroscience providers and reduction in stroke-related deaths.

\section{Methods}

\section{Study Design}

We performed a retrospective analysis of the ARF 2009-2010, a national county-level health information database maintained by the US Department of Health and Human Services. The primary outcome variable was the 3-year (2004-2006) average in stroke deaths per million population for each county. The primary independent variable was the density of neuroscience providers. This was calculated as the total number of neurologists plus neurosurgeons per million population in the year 2006, given the continuum in stroke care from medical to surgical. Data on the numbers of neurologists and neurosurgeons were reported from the American Medical Association Physician Master File to the ARF.

\section{Statistical Analysis}

Multiple linear regression analysis was performed (SAS version 9.2, Windows XP Pro), adjusting for density of GPs (general internal medicine, family medicine, and GPs per million population in the year 2006, reported by the American Medical Association Physician Master File); urbanicity of the county (with rural counties defined as having a rural-urban continuum level $\geq 4$ ); and socioeconomic status of the county (including measures of persistent poverty and low education level as defined by the US Department of Agriculture's Economic Research Service). Counties with persistent poverty are defined as those where $20 \%$ or more of residents were poor as measured by the most recent census. Low-education counties are defined as those where $25 \%$ or more of the residents 25-64 years old had neither a high school diploma nor a General Educational Development diploma. A p value of $<0.05$ was considered to bear statistical significance.

\section{Results}

\section{Descriptive Statistics}

A total of 3141 counties were analyzed. The mean and median number of annual stroke-related deaths per million population was 625 and 586, respectively (SD 275, IQR 449-754). The mean number of neuroscience providers in a county was 21 (SD 52), and the maximum number of neuroscience providers in a county was 1113 , with most of the counties having no neurologists or neurosurgeons (median 0, IQR 0-26). The mean and median number of GPs per million population was 314 and 274, respectively (SD 231, IQR 175-410) (Table 1). Of the counties studied, $2051(65.3 \%)$ were found to have a ruralurban continuum level of $\geq 4$ and were therefore considered rural. Also, 622 counties were coded as having low educational status, and 386 counties were described as having poorer financial status (Table 2).

\section{Predictors of Stroke Deaths}

On unadjusted analysis, each increase of 1 neuroscience provider per million population was associated with 0.71 fewer stroke deaths per million population $(95 \% \mathrm{CI}$ -0.89 to $-0.52, \mathrm{p}<0.001$ ) (Table 3 ). On multivariate analysis, adjusting for urbanicity, socioeconomic status, and GP density, each increase of 1 neuroscience provider per million population was associated with 0.38 fewer stroke deaths per million population $(95 \% \mathrm{CI}-0.57$ to -0.19 , $\mathrm{p}<$ 0.001 ). In contrast, on multivariate analysis, each increase of 1 GP per million population was associated with 0.143 more stroke deaths per million population $(95 \%$ CI 0.102 to $0.184, \mathrm{p}<0.001$ ) (Table 4).

When the effect of other socioeconomic factors on stroke deaths within a county were analyzed, rural location $(\mathrm{p}<0.001)$ was also associated with significant increases in the rate of stroke deaths, whereas persistent poverty and low educational levels were not significant.

\section{Subgroup Analysis}

Additional subset analysis found that the relationship between neuroscience provider density and stroke deaths existed both in rural areas (regression coefficient -0.59 , $95 \% \mathrm{CI}-1.01$ to $-0.18, \mathrm{p}=0.005$ ) (Table 5 ) and in urban areas (regression coefficient $-0.28,95 \% \mathrm{CI}-0.44$ to $-0.11, \mathrm{p}=0.001)($ Table 6).

\section{Discussion}

In the US, stroke is a leading cause of death and dis-

TABLE 1: Descriptive statistics for cerebrovascular disease deaths, neuroscience providers, and GPs*

\begin{tabular}{lccccccc}
\hline \multicolumn{1}{c}{ Variable } & Min & Max & Med & $\begin{array}{c}\text { 25th } \\
\text { Percentile }\end{array}$ & $\begin{array}{c}\text { P5th } \\
\text { Percentile }\end{array}$ & Mean & SD \\
\hline $\begin{array}{l}\text { 3-yr average cerebrovascular disease deaths/ } \\
\text { million population in 2006 }\end{array}$ & 0 & 2432 & 586 & 449 & 754 & 625 & 275 \\
$\begin{array}{l}\text { no. of neuroscience providers/million population } \\
\text { in 2006 }\end{array}$ & 0 & 1113 & 0 & 0 & 26 & 21 & 52 \\
no. of GPs/million population in 2006 & 0 & 3859 & 274 & 175 & 410 & 314 & 231 \\
\hline
\end{tabular}

* The 3-year average included data from 2004 to 2006. Abbreviation: med = median. 
Density of neuroscience providers and stroke deaths

TABLE 2: Frequency and percent for rural county, low educational level, and persistent poverty

\begin{tabular}{|c|c|c|c|c|}
\hline Variable* & Frequency & $\%$ & Cumulative Frequency & Cumulative $\%$ \\
\hline \multicolumn{5}{|l|}{ rural county in 2003} \\
\hline 0 & 1090 & 34.7 & 1090 & 34.7 \\
\hline 1 & 2051 & 65.3 & 3141 & 100 \\
\hline \multicolumn{5}{|c|}{ low education code in 2004} \\
\hline 0 & 2519 & 80.2 & 2519 & 80.2 \\
\hline 1 & 622 & 19.8 & 3141 & 100 \\
\hline \multicolumn{5}{|l|}{ poverty code in 2004} \\
\hline 0 & 2755 & 87.7 & 2755 & 87.7 \\
\hline 1 & 386 & 12.3 & 3141 & 100 \\
\hline
\end{tabular}

* Rural county is defined as having a rural-urban continuum level $\geq 4$.

ability in adults. ${ }^{3-5,17,22,23,26,35}$ The incidence of stroke is estimated at 750,000 cases per year, and has been steadily increasing. ${ }^{29} \mathrm{~A}$ wide variability of stroke prevalence and stroke-related mortality has been observed in different countries, in different states of the US, and even in different counties of the same state. Several investigators have attempted to shed light on this phenomenon. In a global analysis, Johnston et al. ${ }^{21}$ demonstrated that stroke-related mortality was 3.5 times higher in low-income countries than in middle- and high-income countries. The researchers, contrary to what they had initially expected, noticed a lower incidence of cardiovascular risk factors in the poorer countries. Despite this, the lack of appropriate preventive measures in combination with limited access to health care probably contributes to the higher mortality rate observed in lower-income countries.

Within the US, stroke-related mortality appears to vary by race and geographic region. The highest incidence has been reported in the "buckle of the Stroke Belt" (coastal regions of North Carolina, South Carolina, and Georgia) and in the "Stroke Belt states" (remainder of North Carolina, South Carolina, and Georgia, as well as Mississippi, Tennessee, Arkansas, and Louisiana). ${ }^{36}$ Various groups have investigated the association of various factors with this phenomenon. ${ }^{16,25,30,33,36}$ Most studies, however, have focused on factors associated with increased stroke incidence rather than higher case fatality. Lower socioeconomic status, hypertension, and diabetes have most commonly been associated with higher stroke prevalence. Several international studies have been in agreement with these results. ${ }^{2,6,12,18,19}$ In particular, a study from the People's Republic of China ${ }^{18}$ has demonstrated that more than $70 \%$ of the variance in stroke incidence could be accounted for by differences in the prevalence of hypertension. These observations have also been validated by a study in Malmö, Sweden, ${ }^{12}$ an area especially suitable for epidemiological studies, because all the patients are treated in a single academic center. Similar conclusions have been reached by Italian investigators, ${ }^{6}$ who supported that their study was controlled for the disparities in accessibility of care, since their country's healthcare system is publically funded, providing universal access and comprehensive coverage.

The aforementioned studies, with the exception of Cesaroni et al., ${ }^{6}$ have focused almost exclusively on investigating the effect of cardiovascular risk factors on the geographic variations of the incidence and mortality rates of stroke. The potential impact of timely access to specialized care has not been addressed. This is a potentially important (and modifiable) predictor of stroke outcomes. Our study has demonstrated a significant positive effect of increased density of neurologists and neurosurgeons on stroke survival. Although data on other specific risk factors were not available in the ARF, our multivariate regression analysis demonstrated a sustained effect after controlling for socioeconomic status and urbanicity of the county. In the literature, $, 6,12,16,18,25,30,33,36$ there is an established association of lower socioeconomic status with

TABLE 3: Univariate unadjusted analysis for 3-year average cerebrovascular disease deaths in a county*

\begin{tabular}{|c|c|c|c|c|c|}
\hline \multirow[b]{2}{*}{ Variable } & \multirow[b]{2}{*}{ Regression Coefficient } & \multicolumn{2}{|c|}{$95 \% \mathrm{Cl}$} & \multirow[b]{2}{*}{$p$ Value } & \multirow[b]{2}{*}{$\mathrm{R}^{2}$} \\
\hline & & Lower CL & Upper CL & & \\
\hline $\begin{array}{l}\text { no. of neuroscience providers/million } \\
\text { population in } 2006\end{array}$ & -0.71 & -0.89 & -0.52 & $<0.001$ & 0.018 \\
\hline no. of GPs/million population in 2006 & 0.15 & 0.11 & 0.19 & $<0.001$ & 0.016 \\
\hline persistent poverty in 2004 & 18.22 & -11.12 & 47.55 & 0.224 & 0.0005 \\
\hline low educational level in 2004 & 17.58 & -6.58 & 41.75 & 0.154 & 0.0006 \\
\hline rural county in 2003 & 170.99 & 151.65 & 190.32 & $<0.001$ & 0.087 \\
\hline
\end{tabular}

* Unadjusted for urbanicity, low educational level, persistent poverty, neuroscience providers, and GPs. Throughout the tables, negative values denote fewer deaths. 
TABLE 4: Multivariate adjusted analysis for 3-year average cerebrovascular disease deaths in a county

\begin{tabular}{lcrrrr}
\hline & Regression & \multicolumn{2}{c}{$95 \% \mathrm{Cl}$} & \\
\multicolumn{1}{c}{ Variable } & Coefficient & & Lower CL & Upper CL & $\mathrm{p} \mathrm{Value}$ \\
\hline intercept & 488.93 & 468.12 & 509.74 & $<0.001$ \\
no. of neuroscience providers/million population in 2006 & -0.38 & -0.57 & -0.19 & $<0.001$ \\
no. of GPs/million population in 2006 & 0.14 & 0.10 & 0.18 & $<0.001$ \\
persistent poverty in 2004 & -21.07 & -53.21 & 11.06 & 0.199 \\
low educational level in 2004 & 4.38 & -22.33 & 31.09 & 0.748 \\
rural county in 2003 & 155.52 & 134.85 & 176.20 & $<0.001$ \\
\hline
\end{tabular}

* Adjusted for urbanicity, low educational level, persistent poverty, neuroscience providers, and GPs; model $\mathrm{R}^{2}=0.104$

increased cardiovascular risk factors in the US, allowing us to hypothesize that the latter should not be affecting our analysis after controlling for the former. In addition, the present analysis was performed on the county level, recognizing the importance of small-scale variations as underlined by previous studies. ${ }^{30}$

It has been shown ${ }^{1,29}$ that comprehensive stroke centers have a beneficial impact on patient outcomes. A health system can be described as comprising 3 domains: ${ }^{7}$ 1) structure (buildings, equipment, human capital); 2) process (triage, operational algorithm); and 3) outcomes. The contribution of providers both in the structure and the process domains of this model demonstrates their important role in outcomes. Several lines of evidence point to the value of access to appropriate health systems in the outcomes after stroke. ${ }^{1,13,29}$ Along the same lines, telemedicine and telestroke care is increasingly used to provide timely care in stroke patients. ${ }^{8,9,28}$ Recent studies have demonstrated increased stroke-related mortality during the weekends, potentially due to the decreased availability of specialized care providers. ${ }^{20,32}$ The "weekend effect" has been eliminated in comprehensive centers with around-the-clock availability of neurologists, neurosurgeons, and intensivists. ${ }^{1,29}$ This is in agreement with the observed positive effect of more neuroscience providers in decreasing stroke mortality rates in the current analysis.

As demonstrated in our study, there is considerable disparity in the density of neurologists and neurosurgeons practicing among various counties throughout the country. Most counties have no local neurosurgical availability, probably due to a concentration of neurosurgeons around tertiary care centers. The contribution of both specialties, however, in timely interventions is extremely important in stroke, in which emergency care is of paramount importance. Timely diagnosis and treatments supported by significant evidence, such as thrombolysis, ${ }^{27}$ endovascular interventions, ${ }^{15,24,31}$ external ventricular drainage, ${ }^{11}$ and hemicraniectomy, ${ }^{34}$ as well as other options such as hematoma evacuation ${ }^{11}$ in select patients with hemorrhagic stroke are dependent on the immediate availability of neurologists and neurosurgeons.

Our study suggests that an increase of 3 neuroscience providers would be associated with 1 less stroke death per million population. The observed negative effect of primary care providers on stroke mortality rates is difficult to explain. It could potentially be attributed to the decreased density of specialists in counties with a high percentage of primary care providers, or it could be a result of increased recruitment of GPs to high-risk areas. The effect of GPs on primary stroke prevention by risk factor control is probably significant. It appears, however, that stroke-related mortality is mostly dependent on the availability of neurological providers who can provide timely interventions and secondary prevention. In addition, the current study does not analyze the potential effect of neuroscience provider density on stroke morbidity, which may clearly be improved by early diagnosis and intervention. The data in this study demonstrate a public health benefit of neurological and neurosurgical services.

Attempts to improve accessibility to specialized neurological providers locally in underserved areas would probably benefit from multiple strategies, including educational support, medical malpractice reform, and protection of reimbursement, in addition to the development of more effective comprehensive stroke centers in the com-

TABLE 5: Multivariate adjusted analysis for 3-year average cerebrovascular disease deaths in a rural county*

\begin{tabular}{lrrrr}
\hline & Regression & \multicolumn{2}{c}{$95 \% \mathrm{Cl}$} & \\
\cline { 3 - 4 } \multicolumn{1}{c}{ Variable } & Coefficient & Lower CL & Upper CL & p Value \\
\hline intercept & 650.62 & 627.37 & 673.87 & $<0.001$ \\
no. of neuroscience providers/million population in 2006 & -0.59 & -1.01 & -0.18 & 0.005 \\
no. of GPs/million population in 2006 & 0.14 & 0.09 & 0.19 & $<0.001$ \\
persistent poverty in 2004 & -28.08 & -67.97 & 11.80 & 0.167 \\
low educational level in 2004 & -8.50 & -43.37 & 26.38 & 0.633 \\
\hline
\end{tabular}

* Adjusted for low educational level, persistent poverty, neuroscience providers, and GPs; model R² $=0.021$. 
TABLE 6: Multivariate adjusted analysis for 3-year average cerebrovascular disease deaths in an urban county*

\begin{tabular}{lcrrrr}
\hline & Regression & \multicolumn{3}{c}{$95 \% \mathrm{Cl}$} & \\
\multicolumn{1}{c}{ Variable } & Coefficient & & Lower CL & Upper CL & $\mathrm{p} \mathrm{Value}$ \\
\hline intercept & 480.82 & 457.53 & 504.10 & $<0.001$ \\
no. of neuroscience providers/million population in 2006 & -0.28 & -0.44 & -0.11 & 0.001 \\
no. of GPs/million population in 2006 & 0.13 & 0.06 & 0.20 & $<0.001$ \\
persistent poverty in 2004 & 43.39 & -13.18 & 99.96 & 0.133 \\
low educational level in 2004 & 43.19 & 6.39 & 79.99 & 0.021 \\
\hline
\end{tabular}

* Urban county is defined as having rural-urban continuum level 1, 2, or 3 . The analysis was adjusted for low educational level, persistent poverty, neuroscience providers, and GPs; model $\mathrm{R}^{2}=0.024$.

munity. The ACA, which was passed in 2010 , attempts to improve primary care provision by incentivizing primary care at the level of residency training and physician reimbursement. The important role of neurologists and neurosurgeons in decreasing the mortality rate of stroke, the significant contribution of cerebrovascular disease to overall nationwide health care costs, and the scarcity of these providers throughout vast areas of the country, suggest an added importance of promoting vascular neurological practice.

This study has limitations that should be considered. Although an association is demonstrated between neuroscience provider density and reduced stroke-related deaths, a causal relationship cannot be proven by the data. As discussed above, the data do not provide an estimate for the additional effect of neuroscience providers on stroke morbidity, which may be significant. In addition, other markers of the overall quality of the stroke care system (for example specialist equipment and multidisciplinary staffing) were not available in the ARF, and therefore we cannot control for these potential confounders. Furthermore, the data are limited by the accuracy of the initial administrative coding of diagnoses, provider designation, and socioeconomic factors. In addition, the rates of mortality from different types of stroke (for example, ischemic vs hemorrhagic) and specific individual interventions (for example, medical vs surgical) were not available to us, and therefore this study is unable to answer which aspects of stroke management may be most effective in reducing mortality rates. Other individual characteristics such as age and cardiovascular risk factors, which have previously been shown to influence stroke-related mortality, are not considered in our analysis because these data were not available. Another limitation of the current analysis is that not all neurosurgeons and neurologists routinely treat stroke patients, and the data used were not specific to those neuroscience providers involved in stroke care. Finally, the study provides a county-level analysis, which may not always be applicable to individual patients and providers.

\section{Conclusions}

Stroke is a major cause of death in the US. A higher density of neuroscience providers is associated with a significant reduction in deaths from strokes across US counties. This suggests that availability of local neurologists and neurosurgeons may be an important factor in the overall likelihood of survival after stroke, and therefore underlines an importance of promoting specialist clinical neuroscience education and practice throughout the country.

\section{Disclosure}

The authors report no conflict of interest concerning the materials or methods used in this study or the findings specified in this paper.

Author contributions to the study and manuscript preparation include the following. Conception and design: Desai, Bekelis, Ball. Analysis and interpretation of data: Desai. Drafting the article: Desai. Critically revising the article: all authors. Reviewed submitted version of manuscript: all authors. Approved the final version of the manuscript on behalf of all authors: Desai. Statistical analysis: Zhao.

\section{References}

1. Albright KC, Raman R, Ernstrom K, Hallevi H, Martin-Schild $\mathrm{S}$, Meyer BC, et al: Can comprehensive stroke centers erase the 'weekend effect'? Cerebrovasc Dis 27:107-113, 2009

2. Arrich J, Lalouschek W, Müllner M: Influence of socioeconomic status on mortality after stroke: retrospective cohort study. Stroke 36:310-314, 2005

3. Baptista MV, van Melle G, Bogousslavsky J: Prediction of inhospital mortality after first-ever stroke: the Lausanne Stroke Registry. J Neurol Sci 166:107-114, 1999

4. Bogousslavsky J, Van Melle G, Regli F: The Lausanne Stroke Registry: analysis of 1,000 consecutive patients with first stroke. Stroke 19:1083-1092, 1988

5. Brott T, Bogousslavsky J: Treatment of acute ischemic stroke. N Engl J Med 343:710-722, 2000

6. Cesaroni G, Agabiti N, Forastiere F, Perucci CA: Socioeconomic differences in stroke incidence and prognosis under a universal healthcare system. Stroke 40:2812-2819, 2009

7. Chang DC, Eastman B, Talamini MA, Osen HB, Tran Cao HS, Coimbra R: Density of surgeons is significantly associated with reduced risk of deaths from motor vehicle crashes in US counties. J Am Coll Surg 212:862-866, 2011

8. Demaerschalk BM: Seamless integrated stroke telemedicine systems of care: a potential solution for acute stroke care delivery delays and inefficiencies. Stroke 42:1507-1508, 2011

9. Demaerschalk BM, Bobrow BJ, Raman R, Kiernan TE, Aguilar MI, Ingall TJ, et al: Stroke team remote evaluation using a digital observation camera in Arizona: the initial mayo clinic experience trial. Stroke 41:1251-1258, 2010

10. Demaerschalk BM, Hwang HM, Leung G: US cost burden of ischemic stroke: a systematic literature review. Am J Manag Care 16:525-533, 2010

11. Elliott J, Smith M: The acute management of intracerebral 
hemorrhage: a clinical review. Anesth Analg 110:1419-1427, 2010

12. Engström G, Jerntorp I, Pessah-Rasmussen H, Hedblad B, Berglund G, Janzon L: Geographic distribution of stroke incidence within an urban population: relations to socioeconomic circumstances and prevalence of cardiovascular risk factors. Stroke 32:1098-1103, 2001

13. Estabrooks CA, Midodzi WK, Cummings GG, Ricker KL, Giovannetti P: The impact of hospital nursing characteristics on 30-day mortality. J Nurs Adm 41 (7-8 Suppl):S58-S68, 2011

14. Evers SM, Struijs JN, Ament AJ, van Genugten ML, Jager $\mathrm{JH}$, van den Bos GA: International comparison of stroke cost studies. Stroke 35:1209-1215, 2004

15. Fields JD, Lutsep HL, Smith WS, MERCI Multi MERCI Investigators: Higher degrees of recanalization after mechanical thrombectomy for acute stroke are associated with improved outcome and decreased mortality: pooled analysis of the MERCI and Multi MERCI trials. AJNR Am J Neuroradiol 32:2170-2174, 2011

16. Gillum RF, Ingram DD: Relation between residence in the southeast region of the United States and stroke incidence. The NHANES I Epidemiologic Followup Study. Am J Epidemiol 144:665-673, 1996

17. Glass TA, Hennessey PM, Pazdera L, Chang HM, Wityk RJ, Dewitt LD, et al: Outcome at 30 days in the New England Medical Center Posterior Circulation Registry. Arch Neurol 59: 369-376, 2002

18. He J, Klag MJ, Wu Z, Whelton PK: Stroke in the People's Republic of China. I. Geographic variations in incidence and risk factors. Stroke 26:2222-2227, 1995

19. Jakovljević D, Sarti C, Sivenius J, Torppa J, Mähönen M, Immonen-Räihä $\mathrm{P}$, et al: Socioeconomic status and ischemic stroke: The FINMONICA Stroke Register. Stroke 32:14921498, 2001

20. Janszky I, Ahnve S, Ljung R: Weekend versus weekday admission and stroke outcome in Sweden from 1968 to 2005. Stroke 38:e94-e95, 2007 (Letter)

21. Johnston SC, Mendis S, Mathers CD: Global variation in stroke burden and mortality: estimates from monitoring, surveillance, and modelling. Lancet Neurol 8:345-354, 2009

22. Kistler JP: The risk of embolic stroke. Another piece of the puzzle. N Engl J Med 331:1517-1519, 1994

23. Lanska DJ, Kryscio R: Geographic distribution of hospitalization rates, case fatality, and mortality from stroke in the United States. Neurology 44:1541-1550, 1994

24. Lees KR, Bluhmki E, von Kummer R, Brott TG, Toni D, Grotta JC, et al: Time to treatment with intravenous alteplase and outcome in stroke: an updated pooled analysis of ECASS, ATLANTIS, NINDS, and EPITHET trials. Lancet 375:16951703,2010

25. Liao Y, Greenlund KJ, Croft JB, Keenan NL, Giles WH: Factors explaining excess stroke prevalence in the US Stroke Belt. Stroke 40:3336-3341, 2009

26. Libman RB, Kwiatkowski TG, Hansen MD, Clarke WR,
Woolson RF, Adams HP: Differences between anterior and posterior circulation stroke in TOAST. Cerebrovasc Dis 11: 311-316, 2001

27. National Institute of Neurological Disorders and Stroke (NINDS) rt-PA Stroke Study Group: Effect of intravenous recombinant tissue plasminogen activator on ischemic stroke lesion size measured by computed tomography. Stroke 31: 2912-2919, 2000

28. Nelson RE, Saltzman GM, Skalabrin EJ, Demaerschalk BM, Majersik JJ: The cost-effectiveness of telestroke in the treatment of acute ischemic stroke. Neurology 77:1590-1598, 2011

29. O'Brien EC, Rose KM, Shahar E, Rosamond WD: Stroke mortality, clinical presentation and day of arrival: The Atherosclerosis Risk in Communities (ARIC) study. Stroke Res Treat 2011:383012, 2011

30. Pedigo A, Seaver W, Odoi A: Identifying unique neighborhood characteristics to guide health planning for stroke and heart attack: fuzzy cluster and discriminant analyses approaches. PLoS ONE 6:e22693, 2011

31. Penumbra Pivotal Stroke Trial Investigators: The penumbra pivotal stroke trial: safety and effectiveness of a new generation of mechanical devices for clot removal in intracranial large vessel occlusive disease. Stroke 40:2761-2768, 2009

32. Saposnik G, Baibergenova A, Bayer N, Hachinski V: Weekends: a dangerous time for having a stroke? Stroke 38:12111215,2007

33. Siegel PZ, Deeb LC, Wolfe LE, Wilcox D, Marks JS: Stroke mortality and its socioeconomic, racial, and behavioral correlates in Florida. Public Health Rep 108:454-458, 1993

34. Vahedi K, Hofmeijer J, Juettler E, Vicaut E, George B, Algra A, et al: Early decompressive surgery in malignant infarction of the middle cerebral artery: a pooled analysis of three randomised controlled trials. Lancet Neurol 6:215-222, 2007

35. Vemmos KN, Takis CE, Georgilis K, Zakopoulos NA, Lekakis JP, Papamichael CM, et al: The Athens stroke registry: results of a five-year hospital-based study. Cerebrovasc Dis 10:133-141, 2000

36. Voeks JH, McClure LA, Go RC, Prineas RJ, Cushman M, Kissela BM, et al: Regional differences in diabetes as a possible contributor to the geographic disparity in stroke mortality: the REasons for Geographic And Racial Differences in Stroke study. Stroke 39:1675-1680, 2008

Manuscript submitted March 9, 2012.

Accepted October 22, 2012.

Part of this work was presented at the International Stroke Conference, held in New Orleans, Louisiana, in February 2012.

Please include this information when citing this paper: published online November 30, 2012; DOI: 10.3171/2012.10.JNS12518.

Address correspondence to: Atman Desai, M.D., Section of Neurosurgery, Dartmouth-Hitchcock Medical Center, One Medical Center Drive, Lebanon, New Hampshire 03756. email: atman. desai@hitchcock.org. 